\title{
Three Carrier Ambiguity Resolution: Distance-Independent Performance Demonstrated Using Semi-Generated Triple Frequency GPS Signals
}

\author{
Bofeng Li ${ }^{1,2}$ Yanming Feng $^{2}$ Yunzhong Shen ${ }^{1}$ \\ ${ }^{1}$ Department of Surveying and Geo-informatics Engineering, Tongji University, Shanghai 200092, P. R. China \\ ${ }^{2}$ Faculty of Information Technology, Queensland University of Technology, GPO Box 2434, QLD 4001, Australia \\ Email: Bofeng_Li@163.com
}

\begin{abstract}
Despite significant research in development of efficient algorithms for three carrier ambiguity resolution, full performance potential of the additional frequency signals cannot be effectively demonstrated without actual triple frequency data. In addition, all the proposed algorithms showed their difficulties in reliable resolution of the medium-lane and narrow-lane ambiguities in different long-range scenarios. In this contribution, we will investigate the effects of various distance-dependent biases, identifying the tropospheric delay to be the key limitation for long-range three carrier ambiguity resolution. In order to achieve reliable ambiguity resolution in regional networks with the inter-station distances of hundreds of kilometers, a new geometry-free and ionosphere-free model is proposed to fix the integer ambiguities of the medium-lane or narrow-lane observables over just several minutes without distance constraint. Finally, the semi-simulation method is introduced to generate the third frequency signals from dual-frequency GPS data and experimentally demonstrate the research findings of this paper.
\end{abstract}

Keywords GNSS · Three carrier ambiguity resolution $\cdot$ Combination $\cdot$ Success probability

\section{Introduction}

Future Global Navigation Satellite Systems (GNSS) will transmit three or more frequency signals. The L5 (1176.45 $\mathrm{MHz}$ ) signal will be introduced in GPS in addition to the L1 (1575.42 MHz) and L2 (1227.6 MHz). The Galileo system will provide signals centered at E1 (1575.42 MHz), E6 (1278.75 MHz), E5B (1207.14 MHz) and E5A (1176.45 MHz) for commercial and civilian use. The
Chinese COMPASS, the Japanese QZSS and other GNSS systems will also transmit multiple frequency signals. It is anticipated that the efficiency and the reliability of carrier ambiguity resolution for long inter-receiver distance can be significantly enhanced with additional frequency signals, which is rather crucial to realize real-time precise positioning at regional and global scales.

In addressing ambiguity resolution with three frequency signals, the earliest studies by Forssell et al. (1997) and Vollath et al. (1998) described three carrier ambiguity resolution (TCAR) methods. Hatch et al. (2000) proposed the cascading ambiguity resolution (CAR) method. In fact, early TCAR and CAR methods essentially use the same iterative geometry-free approach to estimate integer ambiguities of selected optimal combinations having successively reduced wavelengths. Ji et al. (2007) defined a set of optimal combinations of Galileo inter-frequencies and compared their success probabilities of epochwise ambiguity resolution respectively using CAR and LAMBDA (least squares ambiguity decorrelation adjustment) methods. Henkel and Cünther (2007) investigated the integrity analysis of CAR, where the decorrelation transformation and search technique were applied in each cascading step. Richert and EI-Sheimy (2007) defined useful combinations for the three frequency GPS and Galileo systems which eliminate or mitigate individual error sources, alleviate excessive computational burdens and reduce the communication bandwidth. Feng (2008) proposed a more general geometry-based TCAR strategy which identified three best "virtual" signals to allow more reliable ambiguity resolution under certain observational conditions characterized by ionospheric and tropospheric delays, orbital discrepancy and the level of code and phase noises. The superior performance has been numerically demonstrated in Feng and Li (2008).

Various TCAR methods developed to date could be classified into two basic categories, namely, geometryfree and geometry-based integer determination models. 
The term "geometry-free" refers to the observation model for ambiguity parameters without the geometric distance between receiver and satellite. Geometry-free TCAR directly estimates the float ambiguities from virtual code or virtual ambiguity-fixed phase measurements (Hatch et al. 2000; Feng et al. 2007). Consequently, the total noise level of virtual code and ambiguity-fixed phase must be distinctly smaller than the wavelength for correct ambiguity resolution and the implementation is simple. Unfortunately, this requirement cannot be always satisfied in the medium-lane (ML: $0.19 \mathrm{~m} \leq \lambda<0.75 \mathrm{~m}$ ) and narrowlane (NL: $\lambda<0.19 \mathrm{~m}$ ) ambiguity resolution, particularly in the long-range case. In the geometry-based TCAR one can select three independent optimal combinations from three original signals to form linear equations, and then solve the least squares float ambiguities and conduct the search process using the methods such as LAMBDA, to fix the integers of these combined signals.

The ionospheric effects can be efficiently minimized by selecting the ionosphere-reduced combinations. As a result, the ionosphere would no longer be the key limitation for TCAR over long inter-receiver distances. The question is if there are any more limiting factors in ambiguity resolution over long-distances. Unlike ionospheric effects, the tropospheric effect does not depend on frequency and cannot be cancelled by combination of signals on different frequencies. But it is important to understand in which cases the troposphere will be the limiting factor. In this paper, we will develop a new method that is geometry-free and ionosphere-free to simultaneously overcome the ionospheric and tropospheric effects for reliable ambiguity resolution without distance constraint.

In section 2, we will present the general geometryfree and geometry-based TCAR models for networkbased and/or rover-based ambiguity resolution process. In section 3, the effects of orbital, ionospheric and tropospheric biases are numerically examined for three baselines of different lengths, and the key limiting factor for long-range TCAR is identified. A new geometry-free method without ionospheric effects is proposed in section 4 for reliable ML/NL ambiguity resolution over several minutes without any distance constraints. In the experimental section, we will introduce a semi-simulation method for generating the third frequency signals based on five sets of dual-frequency GPS data from the US Continuously Operating Reference Stations (CORS) network. With these data sets, the performance of geometry-based and geometry-free TCAR models for extra-widelane (EWL: $\lambda \geq 2.93 \mathrm{~m}$ ) ambiguity resolution, along with the new geometry-free and ionosphere-free model for ML/NL ambiguity resolution, are demonstrated.
Research findings in the paper are summarized in the final section.

\section{Geometry-free and geometry-based models for TCAR with distance constraints}

The general geometry-free and geometry-based TCAR models identified by the optimal combinations are systematically analyzed for the different purposes such as network-based and rover-based ambiguity resolution and long baseline ambiguity resolution along with position estimation.

\subsection{Fundamental GNSS combined observational models}

Starting from the combined double differenced (DD) code and phase observation equations,

$$
\begin{aligned}
& \Delta \mathrm{P}_{(\mathrm{i}, \mathrm{j}, \mathrm{k})}=\Delta \rho+\Delta \delta_{\text {orb }}+\Delta \delta_{\text {tro }}+\beta_{(\mathrm{i}, \mathrm{j}, \mathrm{k})} \frac{\Delta \mathrm{K}}{\mathrm{f}_{1}^{2}}+\varepsilon_{\Delta \mathrm{P}_{(\mathrm{i}, \mathrm{j}, \mathrm{k})}} \\
& \Delta \Phi_{(\mathrm{i}, \mathrm{j}, \mathrm{k})}=\Delta \rho+\Delta \delta_{\text {orb }}+\Delta \delta_{\text {tro }}-\beta_{(\mathrm{i}, \mathrm{j}, \mathrm{k})} \frac{\Delta \mathrm{K}}{\mathrm{f}_{1}^{2}} \\
& -\lambda_{(\mathrm{i}, \mathrm{j}, \mathrm{k})} \Delta \mathrm{N}_{(\mathrm{i}, \mathrm{j}, \mathrm{k})}+\varepsilon_{\Delta \Phi_{(\mathrm{i}, \mathrm{j}, \mathrm{k})}}
\end{aligned}
$$

where " $\Delta$ " is the DD operator product. The symbol $\rho$ represents geometric distance from satellite to receiver, $\delta_{\text {orb }}$ is orbital error, $\delta_{\text {tro }}$ is the tropospheric delay, and $\mathrm{K}$ is the parameter of the first-order ionospheric delay. The combined DD code and phase can be expressed as

$$
\begin{aligned}
\Delta \mathrm{P}_{(\mathrm{i}, \mathrm{j}, \mathrm{k})} & =\frac{\mathrm{i} \cdot \mathrm{f}_{1} \cdot \Delta \mathrm{P}_{1}+\mathrm{j} \cdot \mathrm{f}_{2} \cdot \Delta \mathrm{P}_{2}+\mathrm{k} \cdot \mathrm{f}_{5} \cdot \Delta \mathrm{P}_{5}}{\mathrm{i} \cdot \mathrm{f}_{1}+\mathrm{j} \cdot \mathrm{f}_{2}+\mathrm{k} \cdot \mathrm{f}_{5}} \\
\Delta \Phi_{(\mathrm{i}, \mathrm{j}, \mathrm{k})} & =\frac{\mathrm{i} \cdot \mathrm{f}_{1} \cdot \Delta \Phi_{1}+\mathrm{j} \cdot \mathrm{f}_{2} \cdot \Delta \Phi_{2}+\mathrm{k} \cdot \mathrm{f}_{5} \cdot \Delta \Phi_{5}}{\mathrm{i} \cdot \mathrm{f}_{1}+\mathrm{j} \cdot \mathrm{f}_{2}+\mathrm{k} \cdot \mathrm{f}_{5}}
\end{aligned}
$$

where the combination coefficients $\mathrm{i}, \mathrm{j}, \mathrm{k}$ are arbitrary integers; the symbols $\Delta \mathrm{P}_{\mathrm{i}}$ and $\Delta \Phi_{\mathrm{i}}$ are DD code and phase measurements for the ith frequency $f_{i}$. The combined wavelength is defined as

$$
\lambda_{(\mathrm{i}, \mathrm{j}, \mathrm{k})}=\frac{\mathrm{c}}{\mathrm{i} \cdot \mathrm{f}_{1}+\mathrm{j} \cdot \mathrm{f}_{2}+\mathrm{k} \cdot \mathrm{f}_{5}}
$$

and the combined integer ambiguity is

$$
\Delta \mathrm{N}_{(\mathrm{i}, \mathrm{j}, \mathrm{k})}=\mathrm{i} \cdot \Delta \mathrm{N}_{1}+\mathrm{j} \cdot \Delta \mathrm{N}_{2}+\mathrm{k} \cdot \Delta \mathrm{N}_{5}
$$

The combined first-order ionospheric scale factor is defined as 


$$
\beta_{(i, j, k)}=\frac{f_{1}^{2} \cdot\left(i / f_{1}+j / f_{2}+k / f_{5}\right)}{i \cdot f_{1}+j \cdot f_{2}+k \cdot f_{5}}
$$

Assuming that $\quad \sigma_{\Delta P_{1}}=\sigma_{\Delta P_{2}}=\sigma_{\Delta P_{5}} \equiv \sigma_{\Delta P} \quad$ and $\sigma_{\Delta \Phi_{1}}=\sigma_{\Delta \Phi_{2}}=\sigma_{\Delta \Phi_{5}} \equiv \sigma_{\Delta \Phi}$, the variances of combined DD code and phase noises $\varepsilon_{\Delta P_{(i, j, k)}}$ and $\varepsilon_{\Delta \Phi_{(i, j, k)}}$ are given as

$$
\begin{gathered}
\sigma_{\varepsilon_{\Delta p_{(i, j, k)}}^{2}}^{2}=\frac{\left(i \cdot f_{1}\right)^{2}+\left(j \cdot f_{2}\right)^{2}+\left(k \cdot f_{5}\right)^{2}}{\left(i \cdot f_{1}+j \cdot f_{2}+k \cdot f_{5}\right)^{2}} \sigma_{\Delta P}^{2} \equiv \mu_{(i, j, k)}^{2} \sigma_{\Delta P}^{2} \\
\sigma_{\Delta \varepsilon_{(i, j, k)}}^{2}=\frac{\left(i \cdot f_{1}\right)^{2}+\left(j \cdot f_{2}\right)^{2}+\left(k \cdot f_{5}\right)^{2}}{\left(i \cdot f_{1}+j \cdot f_{2}+k \cdot f_{5}\right)^{2}} \sigma_{\Delta \Phi}^{2} \equiv \mu_{(i, j, k)}^{2} \sigma_{\Delta \Phi}^{2}
\end{gathered}
$$

For the brevity of notation, we denote $\Delta \mathrm{K} / \mathrm{f}_{1}^{2}$ by $\Delta \delta \mathrm{I}_{1}$.

\subsection{Geometry-free TCAR models}

The geometry-free process starts with the EWL observable between two closest L-band carriers, for instance, L2 and L5 in the GPS case, using the combined code measurements on the same carriers. The ambiguity is resolved quite reliably by rounding the "float" value to its nearest integer. Traditionally, the wide-lane (WL: $0.75 \mathrm{~m} \leq \lambda<2.93 \mathrm{~m}$ ) combination of two L-band carriers, e.g. L1 and L2, can be resolved also through rounding with assistance of the ambiguity-corrected EWL measurements (Hatch et al. 2000, Feng et al. 2007). With the first two ambiguity-corrected signals, the ambiguity of the third signal, e.g. L1, is resolved by rounding.

In order to obtain the better geometry-free combinations, Feng et al. (2007) provide a general model by using three frequency measurements simultaneously,

$$
\begin{aligned}
\Delta \mathrm{N}_{(\mathrm{i}, \mathrm{j}, \mathrm{k})}= & \frac{\Delta \mathrm{P}_{(\mathrm{l}, \mathrm{m}, \mathrm{n})}-\Delta \Phi_{(\mathrm{i}, \mathrm{j}, \mathrm{k})}}{\lambda_{(\mathrm{i}, \mathrm{j}, \mathrm{k})}}-\frac{\beta_{(\mathrm{l}, \mathrm{m}, \mathrm{n})}+\beta_{(\mathrm{i}, \mathrm{j}, \mathrm{k})}}{\lambda_{(\mathrm{i}, \mathrm{j}, \mathrm{k})}} \Delta \delta \mathrm{I}_{1} \\
& -\frac{\varepsilon_{\Delta \mathrm{P}_{(\mathrm{l}, \mathrm{m}, \mathrm{n})}}-\varepsilon_{\Delta \Phi_{(\mathrm{i}, \mathrm{j}, \mathrm{k})}}}{\lambda_{(\mathrm{i}, \mathrm{j}, \mathrm{k})}}
\end{aligned}
$$

where $(1, m, n)$ and $(i, j, k)$ are generally different sets of integer values, representing possible combinations. Virtual code and phase observables that are minimally affected by the combined ionospheric delay and code and phase noises should be considered as the better choices for ambiguity resolution purpose. For instance, the usual choices, $\quad \Delta \mathrm{P}_{(1,1,0)}-\Delta \Phi_{(1,-1,0)}$ and $\Delta \mathrm{P}_{(1,0,1)}-\Delta \Phi_{(1,0,-1)}$ for WL ambiguity resolution and $\Delta \mathrm{P}_{(0,1,1)}-\Delta \Phi_{(0,1,1)}$ for EWL ambiguity resolution, are free of ionospheric effects and nearly minimally effected by code noises.
In principle, the geometry-free model (10) can be used to estimate the ML/NL ambiguities. The problem is the enlarged effect of the total noise including the ionospheric delay and code and phase noises in the category of ML/NL signals that must be linearly independent with the first two combinations. In order to overcome this problem, we turn to an ambiguity-corrected WL signals with a relatively smaller total noise level, e.g. $\Delta \Phi_{(1,-1,0)}$, to determine the ML/NL ambiguity. The equation is given as follows,

$$
\begin{aligned}
\Delta \mathrm{N}_{(\mathrm{i}, \mathrm{j}, \mathrm{k})}= & \frac{\Delta \tilde{\Phi}_{(\mathrm{l},-1,0)}-\Delta \Phi_{(\mathrm{i}, \mathrm{j}, \mathrm{k})}}{\lambda_{(\mathrm{i}, \mathrm{j}, \mathrm{k})}}+\frac{\beta_{(1,-1,0)}-\beta_{(\mathrm{i}, \mathrm{j}, \mathrm{k})}}{\lambda_{(\mathrm{i}, \mathrm{j}, \mathrm{k})}} \Delta \delta \mathrm{I}_{1} \\
& -\frac{\varepsilon_{\Delta \Phi_{(1,-1,0)}}-\varepsilon_{\Delta \Phi_{(i, j, k)}}}{\lambda_{(\mathrm{i}, \mathrm{j}, \mathrm{k})}}
\end{aligned}
$$

where $\Delta \tilde{\Phi}_{(1,-1,0)}=\Delta \Phi_{(1,-1,0)}+\lambda_{(1,-1,0)} \Delta \mathrm{N}_{(1,-1,0)}$ is ambiguitycorrected WL observable. In this situation, although the joint random noise of code and phase can be significantly mitigated, the residual ionospheric delay can bias the estimator in the long-range scenario. As a result, the reliable ML/NL ambiguity resolution is not possible unless the ionospheric delay in (11) is efficiently corrected.

\subsection{Geometry-based TCAR models}

In rover-end RTK processing, ambiguities are fixed along with position estimation, where the geometry-based model is usually used. For several triple frequency cases, Feng (2008) identified the three most useful combinations for each of the three frequency GNSS services based on the total noise level in cycles including the effects of orbital, ionospheric and tropospheric biases and phase noises,

$$
\sigma_{\mathrm{TC}}=\frac{\sqrt{\left(\beta_{(\mathrm{i}, \mathrm{j}, \mathrm{k})} \Delta \delta \mathrm{I}_{1}\right)^{2}+\Delta \delta_{\mathrm{tro}}^{2}+\Delta \delta_{\mathrm{orb}}^{2}+\mu_{(\mathrm{i}, \mathrm{j}, \mathrm{k})}^{2} \sigma_{\Delta \Phi}^{2}}}{\lambda_{(\mathrm{i}, \mathrm{j}, \mathrm{k})}}=\min (
$$

with $\sigma_{\mathrm{TC}}$ being the total noise level in cycles. Any combination can be considered being a particular choice according to a certain set of error budgets in the total noise level. Given three sets of error budgets, typically representing the short, medium and long baselines respectively with lengths $d$ satisfying $d \leq 100 \mathrm{~km}$, $100 \mathrm{~km}<\mathrm{d} \leq 200 \mathrm{~km}$ and $\mathrm{d} \geq 200 \mathrm{~km}$, Table 1 summarizes the total noise level in cycles for the most useful combined observables in two categories: (I) EWLs and WLs in the rows 2 to 5 and (II) MLs and NLs in the rows 6 to 11. For a complete TCAR process, one usually chooses two 
observables from category (I) and one from category (II). It is observed that the total noise level for EWL $\Delta \Phi_{(1,-6,5)}$ is 0.160 to 0.174 cycles even under the effects of large orbital, ionospheric and tropospheric biases in the long baseline assumption. This implies that ambiguity resolution of the EWL $\Delta \Phi_{(1,-6,5)}$ using the geometry-based model can be fairly reliable over long distances. Its ambiguity resolution success probability has been theoretically predicted to exceed 99\% with epochwise measurements in long range scenarios. Along with the EWL signals $\Delta \Phi_{(0,1,-1)}$ preferably resolved with the geometry-free model (10), the DD ionospheric bias can be estimated and smoothed with $3 \mathrm{~cm}$ accuracy over about 2 min (Feng and Li 2008).

In all cases, the chosen virtual signals often have reduced ionospheric effects relative to their wavelengths. It is important to notice that the geometry-based model cannot reduce the tropospheric delay at all.

Table 1 The total noise level in cycles for geometry-based optimal combinations under the different given error budgets

\begin{tabular}{rccc}
\hline & \multicolumn{3}{c}{ Total noise level $\sigma_{\text {TC }}$ (in cycles) } \\
\cline { 2 - 4 } virtual & \multicolumn{3}{c}{$\sigma_{\Delta \Phi}=5 \mathrm{~mm}$} \\
signals & $\Delta \delta \mathrm{I}_{1}=10 \mathrm{~cm}$ & $\Delta \delta \mathrm{I}_{1}=20 \mathrm{~cm}$ & $\Delta \delta \mathrm{I}_{1}=100 \mathrm{~cm}$ \\
& $\Delta \delta_{\text {tro }}=5 \mathrm{~cm}$ & $\Delta \delta_{\text {tro }}=10 \mathrm{~cm}$ & $\Delta \delta_{\text {tro }}=15 \mathrm{~cm}$ \\
& $\Delta \delta_{\text {orb }}=1 \mathrm{~cm}$ & $\Delta \delta_{\text {orb }}=2 \mathrm{~cm}$ & $\Delta \delta_{\text {orb }}=8 \mathrm{~cm}$ \\
\hline$\Delta \Phi_{(0,1,-1)}$ & 0.042 & 0.067 & 0.297 \\
$\Delta \Phi_{(1,-6,5)}$ & 0.160 & 0.163 & 0.174 \\
$\Delta \Phi_{(1,-5,4)}$ & 0.138 & 0.154 & 0.358 \\
$\Delta \Phi_{(1,-1,0)}$ & 0.164 & 0.322 & 1.510 \\
$\Delta \Phi_{(1,0,0)}$ & 0.590 & 1.180 & 5.376 \\
$\Delta \Phi_{(2,-1,0)}$ & 0.502 & 0.999 & 4.012 \\
$\Delta \Phi_{(2,0,-1)}$ & 0.486 & 0.968 & 3.752 \\
$\Delta \Phi_{(4,-3,0)}$ & 0.468 & 0.913 & 2.044 \\
$\Delta \Phi_{(4,0,-3)}$ & 0.487 & 0.951 & 1.998 \\
$\Delta \Phi_{(5,-4,0)}$ & 0.532 & 1.031 & 2.249 \\
\hline
\end{tabular}

Table 2 The total noise level in meters for code and ambiguitycorrected phase combinations under the different given error budgets

\begin{tabular}{rccc}
\hline & \multicolumn{3}{c}{ Total noise level $\sigma_{\mathrm{TM}}$ (in meters) } \\
virtual & \multicolumn{3}{c}{$\sigma_{\Delta \mathrm{P}}=50 \mathrm{~cm} / \sigma_{\Delta \Phi}=0.5 \mathrm{~cm}$} \\
\cline { 2 - 4 } signals & $\Delta \delta \mathrm{I}_{1}=10 \mathrm{~cm}$ & $\Delta \delta \mathrm{I}_{1}=20 \mathrm{~cm}$ & $\Delta \delta \mathrm{I}_{1}=100 \mathrm{~cm}$ \\
& $\Delta \delta_{\text {tro }}=5 \mathrm{~cm}$ & $\Delta \delta_{\text {tro }}=10 \mathrm{~cm}$ & $\Delta \delta_{\text {tro }}=20 \mathrm{~cm}$ \\
$\Delta \delta_{\text {orb }}=1 \mathrm{~cm}$ & $\Delta \delta_{\text {orb }}=2 \mathrm{~cm}$ & $\Delta \delta_{\text {orb }}=8 \mathrm{~cm}$ \\
\hline$\Delta \mathrm{P}_{(1,1,1)}$ & 0.329 & 0.421 & 1.479 \\
$\Delta \mathrm{P}_{(1,0,0)}$ & 0.512 & 0.548 & 1.139 \\
$\Delta \mathrm{P}_{(1,1,0)}$ & 0.382 & 0.451 & 1.349 \\
$\Delta \mathrm{P}_{(77,-60,0)}$ & 1.490 & 1.493 & 1.505 \\
$\Delta \tilde{\Phi}_{(1,-1,0)}$ & 0.141 & 0.278 & 1.306 \\
$\Delta \tilde{\Phi}_{(1,0,-1)}$ & 0.246 & 0.393 & 1.741 \\
$\Delta \tilde{\Phi}_{(1,-6,5)}$ & 0.521 & 0.531 & 0.568 \\
$\Delta \tilde{\Phi}_{(1,-5,4)}$ & 0.289 & 0.322 & 0.749 \\
\hline
\end{tabular}

In geometry-based model, we must introduce at least three code or ambiguity-corrected phase DD measurements to provide the coordinate estimates and avoid a rank defect. Lower noised code or ambiguitycorrected phase measurements provide stronger constraints to the integer estimation. One can identify the optimal code or ambiguity-corrected phase observables with the criterion similar to (12) for position solution,

$\sigma_{\mathrm{TM}}=\sqrt{\left(\beta_{(\mathrm{i}, \mathrm{j}, \mathrm{k})} \Delta \delta \mathrm{I}_{1}\right)^{2}+\Delta \delta_{\text {tro }}^{2}+\Delta \delta_{\text {orb }}^{2}+\mu_{(\mathrm{i}, \mathrm{j}, \mathrm{k})}^{2} \sigma_{\Delta \mathrm{P}}^{2}}=\min$

The symbol $\sigma_{\mathrm{TM}}$ denotes the total noise level in meters; $\sigma_{\Delta \mathrm{P}}$ is replaced by $\sigma_{\Delta \Phi}$ if the ambiguity-corrected phase measurements are used. Referring to the three sets of error budgets presented in Table 1, Table 2 outlines the total noise levels in meters for four code and four ambiguitycorrected phase measurements. It is observed that the ambiguity-corrected phase measurements are, in general, better than code measurements. For code measurements, $\Delta \mathrm{P}_{(1,1,1)}$ and $\Delta \mathrm{P}_{(1,1,0)}$ are the best or the geometry-based ambiguity resolution in short and medium baselines. For long baselines, the original code $\Delta \mathrm{P}_{(1,0,0)}$ would be a better choice, and the ionosphere-free code $\Delta \mathrm{P}_{(77,-60,0)}$ provides the stronger constraint when the effect of ionospheric delay becomes rather severe.

\section{Limiting factors in long-range TCAR scenarios}

Ionosphere is traditionally the key limitation to the longrange ambiguity resolution with single and double frequency measurements. This is no longer the case with triple frequency measurements; the key limitation now is the troposphere. This conclusion is evident from the numerical comparison between the effects of ionospheric and tropospheric biases on TCAR along with the orbital errors.

First the discrepancies and uncertainties of GPS broadcast ephemeris are assessed through comparing with International GNSS Services (IGS) final orbit products of 24 hours on January 20, 2008. Differences are mostly smaller than $4 \mathrm{~m}$ as illustrated in Figure 1 where the colors, blue, green and red, represent three coordinate components. The root mean squares (RMS) of broadcast ephemeris errors for all available satellites are illustrated in Figure 2, showing the overall three-dimensional (3D) RMS of better than $3 \mathrm{~m}$. The effect of this 3D RMS orbital error on single differenced baseline over $100 \mathrm{~km}$ is estimated to be smaller than $1.5 \mathrm{~cm}$ and much smaller 
after double differencing (Leick 2004). Moreover, the ultra-rapid predicted precise orbits are available in real time with 3D RMS of $10 \mathrm{~cm}$. Thus the orbital effect is not of concern for long-range application.

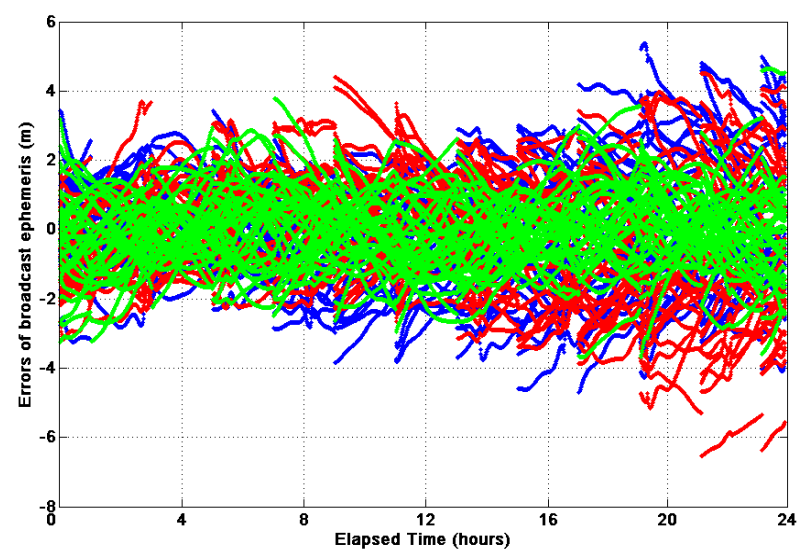

Figure 1 Differences of satellite coordinates from broadcast and precise ephemeris

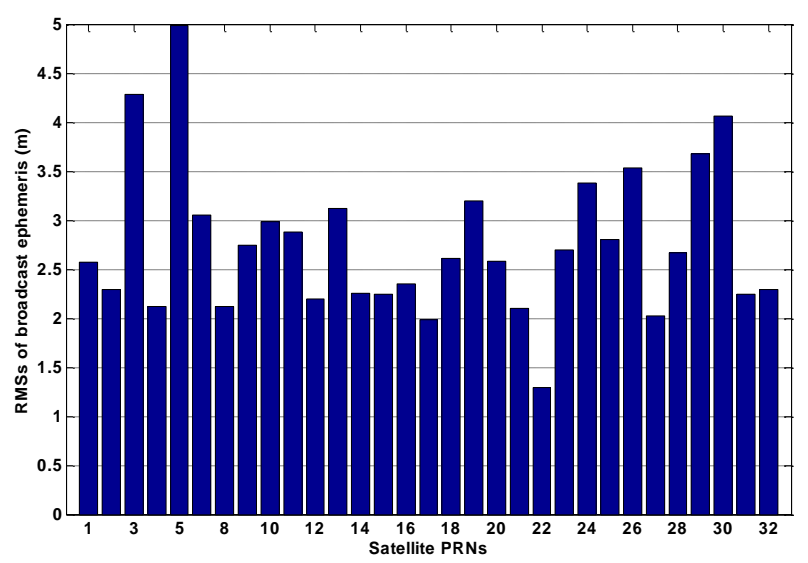

Figure 2 RMSs of broadcast ephemeris for all satellites

In the following, the effects of ionospheric and tropospheric biases are numerically examined for three baselines of different lengths and the mask elevation is set to 15 degrees for all computation. Figure 3 presents the DD ionospheric delays of three baselines with $53 \mathrm{~km}, 78$ $\mathrm{km}$ and $155 \mathrm{~km}$ in length; the magnitudes reach up to 20 $\mathrm{cm}, 30 \mathrm{~cm}$ and $50 \mathrm{~cm}$ respectively. The contributions to the total noise in cycles on L1 carrier would be 1.0, 1.5 and 2.5 respectively. This is why the ionospheric delay is traditionally considered the key limitation to long-range single and double frequency ambiguity resolution. However, this situation is different if third frequency signals are considered. Referring to subsections 2.2 and 2.3, the effects of ionospheric delays in geometry-free and geometry-based TCAR models are either eliminated or significantly reduced. For example, EWL $\Delta \Phi_{(1,-6,5)}$, NLs $\Delta \Phi_{(4,-3,0)}$ and $\Delta \Phi_{(4,0,-3)}$ have the ionospheric scale factors of $-0.0744,0.0902$ and -0.0099 respectively, and thus they are insensitive to ionospheric delays in long-range situation.
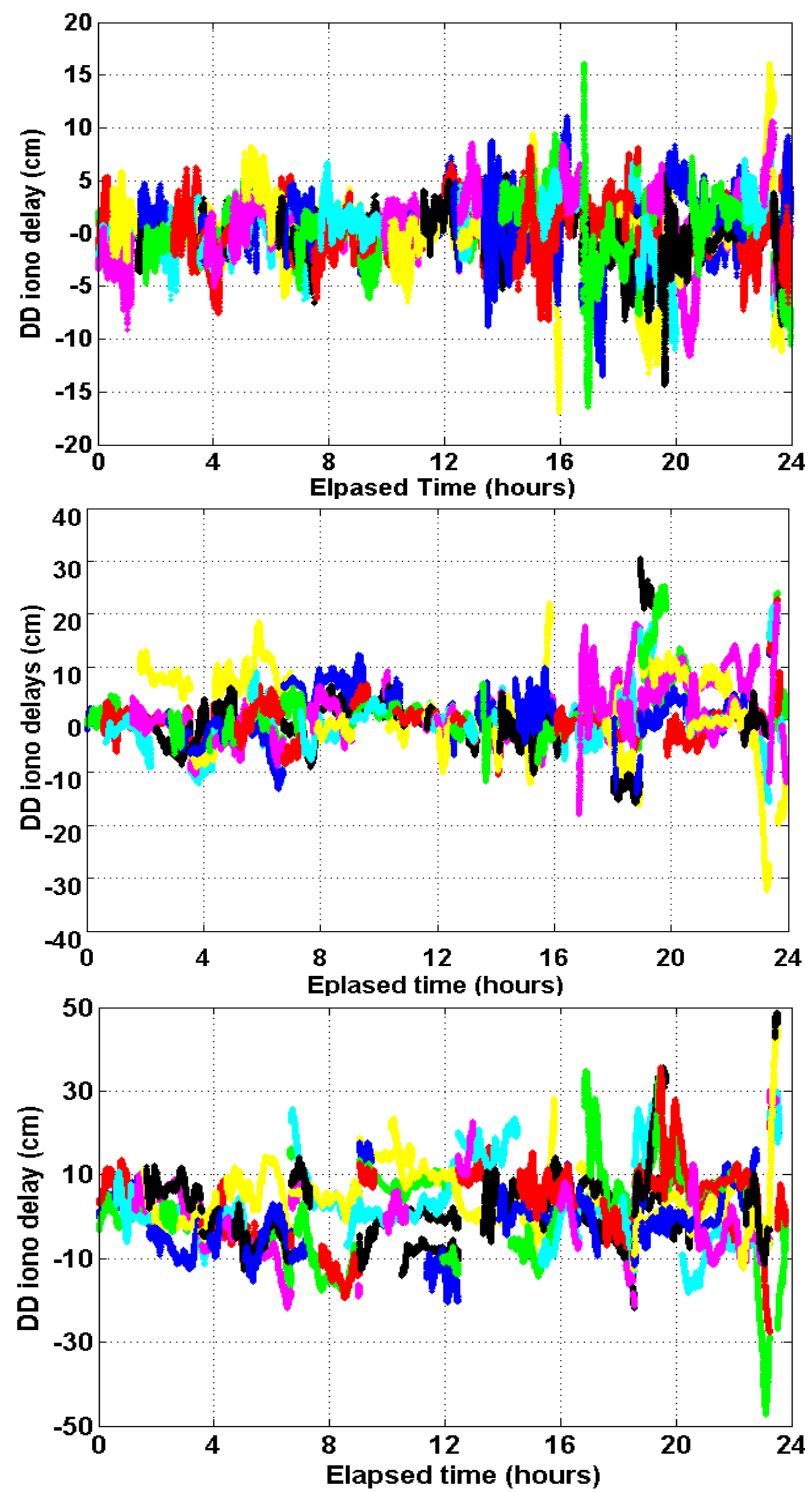

Figure 3 DD ionospheric delays for three baselines (length of baselines: upper $53 \mathrm{~km}$; middle $78 \mathrm{~km}$; lower $155 \mathrm{~km}$ )

Although the ionospheric delay can be efficiently mitigated in the NL combinations, the long-range TCAR does not become easier because of the tropospheric effect. In the computation of residual DD tropospheric biases, we use the UNB3 model to correct the one-way tropospheric delay where all the meteorological parameters are 
interpolated according to the latitude and level height with taking the seasonal variation into account. For more information, one can refer to Collins (1999). Figure 4 shows the residual DD tropospheric biases with $10 \mathrm{~cm}, 18$ $\mathrm{cm}$ and $25 \mathrm{~cm}$ in magnitudes for the baselines of $53 \mathrm{~km}$, $78 \mathrm{~km}$ and $155 \mathrm{~km}$. Although the residual DD tropospheric delays are generally smaller than their corresponding DD ionospheric delays, they cannot be eliminated or reduced through combination operation. Therefore, the tropospheric effect is the key limitation to long-range TCAR performance. However, the geometryfree model may be employed to avoid the effects of troposphere on ML/NL ambiguity resolution in longrange cases.
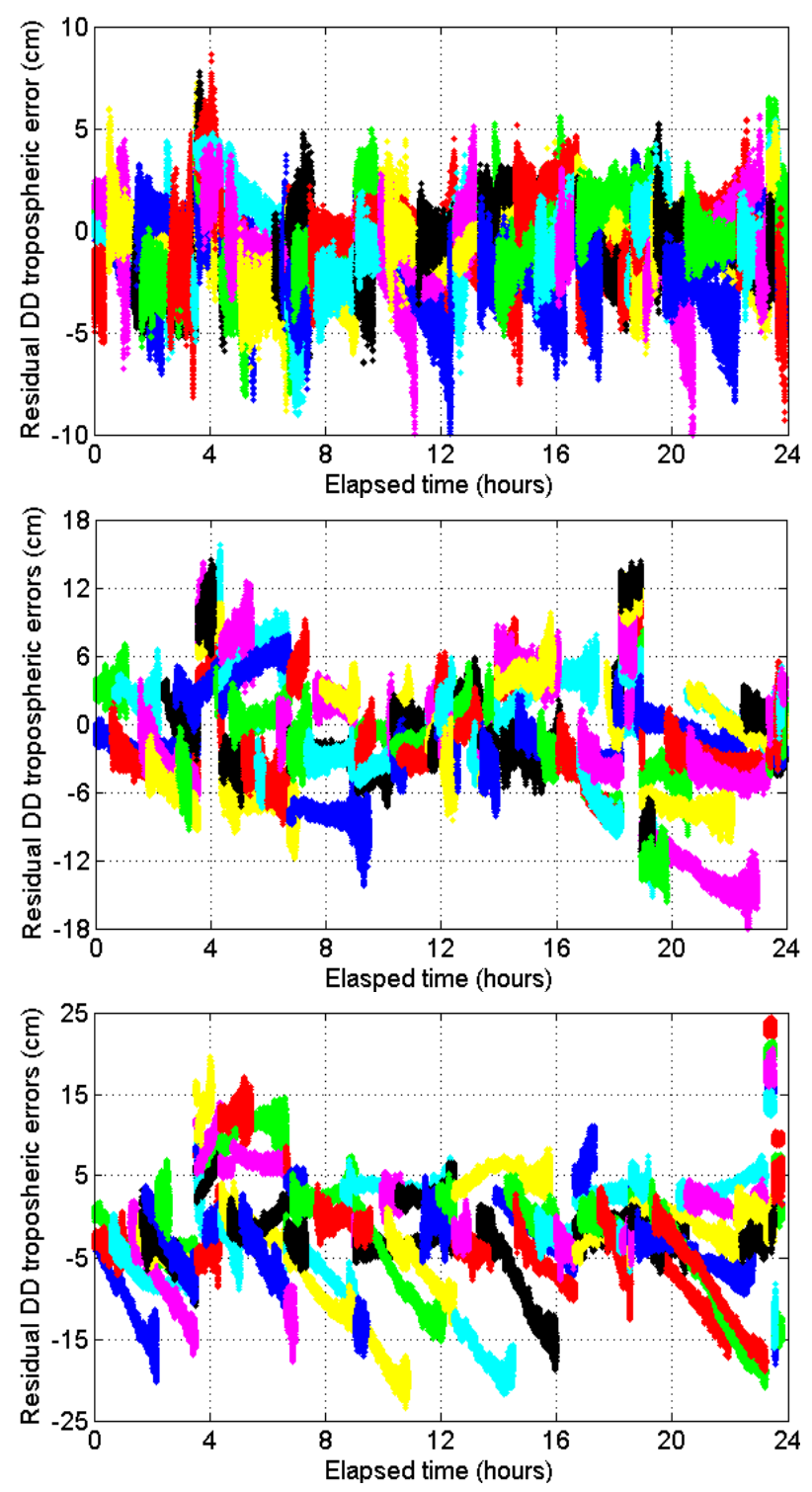

Figure 4 DD tropospheric delays for three baselines (lengths of baselines: upper $53 \mathrm{~km}$; middle $78 \mathrm{~km}$; lower $155 \mathrm{~km}$ )
4. Geometry-free and ionosphere-free model for distance-independent reliable TCAR

The geometry-free and ionosphere-free model is free of both ionospheric effects and geometric terms. Incorrect ambiguity resolution in the network-based process will lead to wrong user position. Ideally, with triple frequency it is possible to overcome the effects of varying ionospheric and tropospheric biases through forming a geometry-free and ionosphere-free function. It is noticed that the EWL ambiguities are rather easy to fix and only two are independent amongst all EWL and WL combinations, and all others can be derivatives of these two signals. For example, the WL ambiguity $\Delta \mathrm{N}_{(1,-1,0)}$ is determined from two EWLs, $\Delta \mathrm{N}_{(0,1,-1)}$ and $\Delta \mathrm{N}_{(1,-6,-5)}$, as

$$
\Delta \mathrm{N}_{(1,-1,0)}=5 \Delta \mathrm{N}_{(0,1,-1)}+\Delta \mathrm{N}_{(1,-6,5)}
$$

In fact, the ambiguity-corrected EWL observables can be considered as code observables with their higher accuracies. Thus they can be directly used to realize decimeter positioning over several hundred kilometers with assistance of filtering (Feng and Li 2008).

Once we have fixed two EWL/WL ambiguities, the geometry-free and ionosphere-free combination is formed using their ambiguity-corrected values, $\Delta \tilde{\Phi}_{(1, \mathrm{~m}, \mathrm{n})}$ and $\Delta \tilde{\Phi}_{(\mathrm{p}, \mathrm{q}, \mathrm{r})}$, and one ML/NL observable $\Delta \Phi_{(\mathrm{i}, \mathrm{j}, \mathrm{k})}$. The ML/NL ambiguity $\Delta \mathrm{N}_{(\mathrm{i}, \mathrm{j}, \mathrm{k})}$ is estimated by

$$
\Delta \mathrm{N}_{(\mathrm{i}, \mathrm{j}, \mathrm{k})}=\frac{\mathrm{a}_{1} \Delta \tilde{\Phi}_{(1, \mathrm{~m}, \mathrm{n})}+\mathrm{a}_{2} \Delta \tilde{\Phi}_{(\mathrm{p}, \mathrm{q}, \mathrm{r})}-\Delta \Phi_{(\mathrm{i}, \mathrm{j}, \mathrm{k})}}{\lambda_{(\mathrm{i}, \mathrm{j}, \mathrm{k})}}-\varepsilon_{\Delta \mathrm{N}_{(\mathrm{i}, \mathrm{j}, \mathrm{k})}}
$$

with the total noise of

$$
\varepsilon_{\Delta \mathrm{N}_{(\mathrm{i}, \mathrm{j}, \mathrm{k})}}=\frac{\mathrm{a}_{1} \varepsilon_{\Delta \Phi_{(\mathrm{l}, \mathrm{m}, \mathrm{n})}}+\mathrm{a}_{2} \varepsilon_{\Delta \Phi_{(\mathrm{p}, \mathrm{q}, \mathrm{r})}}-\varepsilon_{\Delta \Phi_{(\mathrm{i}, \mathrm{j}, \mathrm{k})}}}{\lambda_{(\mathrm{i}, \mathrm{j}, \mathrm{k})}}
$$

According to the condition of being both geometry-free and ionosphere-free, the coefficients are determined by

$$
\left.\begin{array}{c}
\mathrm{a}_{1} \beta_{(1, \mathrm{~m}, \mathrm{n})}+\mathrm{a}_{2} \beta_{(\mathrm{p}, \mathrm{q}, \mathrm{r})}=\beta_{(\mathrm{i}, \mathrm{j}, \mathrm{k})} \\
\mathrm{a}_{1}+\mathrm{a}_{2}=1
\end{array}\right\}
$$

Thus

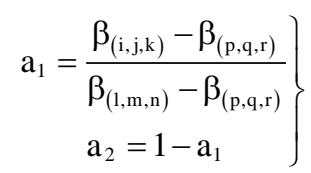

The standard deviation of the ML/NL ambiguity estimator is calculated in cycles by 


$$
\sigma_{\Delta \mathrm{N}_{(\mathrm{i}, \mathrm{j}, \mathrm{k})}}=1013.5 \sigma_{\Delta \Phi}
$$

for arbitrary choices of EWL/WL and ML/NL observables as shown in Table 3. Apparently, the ambiguity resolution performance for all schemes is equivalent, and the standard deviation of estimated ambiguity is 5.068 and 10.135 cycles respectively for $\sigma_{\Delta \Phi}=0.5$ and $1 \mathrm{~cm}$. It is noticed that the ionospheric and tropospheric effects are totally cancelled. Therefore, ML/NL ambiguity resolution is distance-independent and can be efficiently achieved by rounding the averaged float ambiguities in the time domain over arbitrary long distances.

Referring to subsection 2.2, if reliable and rapid geometry-free TCAR over short-distance is of concern, one can choose two EWL combinations $\Delta \mathrm{P}_{(0,1,1)}-\Delta \Phi_{(0,1,-1)}$, $\Delta \mathrm{P}_{(1,1,0)}-\Delta \Phi_{(1,-4,3)}$ and one $\mathrm{ML}$ combination $\Delta \tilde{\Phi}_{(1,-1,0)}-\Delta \Phi_{(1,0,0)}$ to fix all the integer ambiguities with measurements of a few epochs without ionospheric corrections. In case of the regional CORS network where the inter-station distances range up to hundreds of kilometers, the selections can include $\Delta \mathrm{P}_{(0,1,1)}-\Delta \Phi_{(0,1,-1)}$, $\Delta \mathrm{P}_{(1,1,0)}-\Delta \Phi_{(1,-1,0)}$ and any one scheme in Table 3 with (15), where all the distance-dependent terms cancel. The integers can be determined simply and reliably by averaging and rounding operations; the approach therefore is very suitable for the network-based process without inter-receiver distance constraint.

Table 3 Geometry-free and ionosphere-free combinations for reliable ML/NL ambiguity resolution

\begin{tabular}{ccccc}
\hline \multirow{2}{*}{$\Delta \tilde{\Phi}_{(1, \mathrm{~m}, \mathrm{n})}$} & $\Delta \tilde{\Phi}_{(\mathrm{p}, \mathrm{q}, \mathrm{r})}$ & $\Delta \Phi_{(\mathrm{i}, \mathrm{j}, \mathrm{k})}$ & \multicolumn{2}{c}{$\sigma_{\Delta \mathrm{N}_{(\mathrm{i}, \mathrm{j}, \mathrm{k})}}$ (in cycles) } \\
\cline { 4 - 5 } & & & $\sigma_{\Delta \Phi}=5 \mathrm{~mm}$ & $\sigma_{\Delta \Phi}=1 \mathrm{~cm}$ \\
\hline$\Delta \Phi_{(0,1,-1)}$ & $\Delta \Phi_{(1,-6,5)}$ & $\Delta \Phi_{(1,0,0)}$ & 5.068 & 10.135 \\
$\Delta \Phi_{(0,1,-1)}$ & $\Delta \Phi_{(1,-6,5)}$ & $\Delta \Phi_{(0,1,0)}$ & 5.068 & 10.135 \\
$\Delta \Phi_{(0,1,-1)}$ & $\Delta \Phi_{(1,-6,5)}$ & $\Delta \Phi_{(0,0,1,)}$ & 5.068 & 10.135 \\
$\Delta \Phi_{(0,1,-1)}$ & $\Delta \Phi_{(1,-6,5)}$ & $\Delta \Phi_{(4,-1,-2)}$ & 5.068 & 10.135 \\
$\Delta \Phi_{(0,1,-1)}$ & $\Delta \Phi_{(1,-6,5)}$ & $\Delta \Phi_{(4,-3,0)}$ & 5.068 & 10.135 \\
$\Delta \Phi_{(0,1,-1)}$ & $\Delta \Phi_{(1,-6,5)}$ & $\Delta \Phi_{(4,0,-3)}$ & 5.068 & 10.135 \\
$\Delta \Phi_{(1,-1,0)}$ & $\Delta \Phi_{(1,0,-1)}$ & $\Delta \Phi_{(1,0,0)}$ & 5.068 & 10.135 \\
$\Delta \Phi_{(1,-1,0)}$ & $\Delta \Phi_{(1,0,-1)}$ & $\Delta \Phi_{(0,1,0)}$ & 5.068 & 10.135 \\
$\Delta \Phi_{(1,-1,0)}$ & $\Delta \Phi_{(1,0,-1)}$ & $\Delta \Phi_{(0,0,1)}$ & 5.068 & 10.135 \\
$\Delta \Phi_{(1,-1,0)}$ & $\Delta \Phi_{(1,0,-1)}$ & $\Delta \Phi_{(4,-1,-2)}$ & 5.068 & 10.135 \\
$\Delta \Phi_{(1,-1,0)}$ & $\Delta \Phi_{(1,0,-1)}$ & $\Delta \Phi_{(4,-3,0)}$ & 5.068 & 10.135 \\
$\Delta \Phi_{(1,-1,0)}$ & $\Delta \Phi_{(1,0,-1)}$ & $\Delta \Phi_{(4,0,-3)}$ & 5.068 & 10.135 \\
\hline
\end{tabular}

\section{Experiment and analysis}

In this section, we will introduce a semi-simulation method proposed by $\mathrm{Li}$ (2008) for generating the third frequency signals based on existent dual-frequency GPS measurements to demonstrate the full TCAR potential. The semi-generated data can retain the consistent systematic biases with the real world situation. The method includes three main steps: (i) separate the DD ionospheric and tropospheric biases based on the fixed L1 and L2 ambiguities; (ii) assess the stochastic characteristics of double frequency phase and code measurements, involving accuracy and cross correlation between L1 and L2 phases, to quantify the noise terms added to the third frequency signals; (iii) generate the third frequency code and phase signals using the estimated ionospheric and tropospheric biases and stochastic characteristics. Five 24-hour GPS data sets were collected from the US CORS (http://www.ngs.noaa.gov/CORS) on February 12008. All data were sampled with 1 second and the mask angle of 15 degrees was set in the processing. The five baseline lengths in units of kilometers are 53, 78, 98, 120 and 155. For each baseline, the third DD code (P5) and DD phase (L5) are semi-generated.

Table 4 Success probability of ambiguity resolution for some frequently used optimal combinations in geometry-free or geometry-based models

\begin{tabular}{cccccc}
\hline \multirow{2}{*}{ models } & \multicolumn{5}{c}{ Success probability (\%) } \\
\cline { 2 - 6 } & $53 \mathrm{~km}$ & $78 \mathrm{~km}$ & $98 \mathrm{~km}$ & $120 \mathrm{~km}$ & $155 \mathrm{~km}$ \\
\hline$\Delta \mathbf{P}_{(\mathbf{0 , 1 , 1})}-\Delta \boldsymbol{\Phi}_{(\mathbf{0 , 1 , - 1})}$ & $\mathbf{1 0 0 . 0}$ & $\mathbf{1 0 0 . 0}$ & $\mathbf{1 0 0 . 0}$ & $\mathbf{1 0 0 . 0}$ & $\mathbf{1 0 0 . 0}$ \\
$\Delta \mathrm{P}_{(1,1,0)}-\Delta \Phi_{(1,-6,5)}$ & 99.40 & 100.0 & 100.0 & 99.99 & 99.98 \\
$\Delta \mathrm{P}_{(1,1,0)}-\Delta \Phi_{(1,-5,4)}$ & 99.30 & 99.95 & 99.98 & 99.88 & 99.73 \\
$\Delta \mathrm{P}_{(1,1,0)}-\Delta \Phi_{(1,-1,0)}$ & 90.80 & 92.71 & 93.95 & 90.08 & 91.35 \\
$\Delta \boldsymbol{\Phi}_{(\mathbf{1},-\mathbf{6}, \mathbf{5})}, \Delta \mathbf{P}_{(\mathbf{1}, \mathbf{1}, \mathbf{0})}$ & $\mathbf{9 9 . 9 9}$ & $\mathbf{1 0 0 . 0}$ & $\mathbf{1 0 0 . 0}$ & $\mathbf{1 0 0 . 0}$ & $\mathbf{1 0 0 . 0}$ \\
$\Delta \Phi_{(1,-5,4)}, \Delta \mathrm{P}_{(1,1,0)}$ & 99.96 & 100.0 & 100.0 & 100.0 & 99.99 \\
$\Delta \Phi_{(1,-6,5)}, \Delta \mathrm{P}_{(77,-60,0)}$ & 99.09 & 99.03 & 95.72 & 98.99 & 97.00 \\
$\Delta \Phi_{(1,-5,4)}, \Delta \mathrm{P}_{(77,-60,0)}$ & 98.23 & 95.59 & 95.18 & 94.56 & 94.40 \\
$\Delta \Phi_{(4,0,-3)}, \Delta \tilde{\Phi}_{(1,0,-1)}$ & 83.33 & 36.42 & 24.04 & 26.41 & 22.70 \\
$\Delta \boldsymbol{\Phi}_{(\mathbf{4 , - 3 , 0 )},}, \Delta \tilde{\Phi}_{(1,-1,0)}$ & $\mathbf{8 3 . 3 3}$ & $\mathbf{3 7 . 1 1}$ & $\mathbf{2 4 . 9 4}$ & $\mathbf{2 8 . 2 0}$ & $\mathbf{2 4 . 4 5}$ \\
\hline
\end{tabular}

The ambiguity resolution potentials of optimal combinations extensively used in general geometry-free or geometry-based models are evaluated from single 
epoch measurements first. We define the success probability to be the ratio of the number of epochs with all correct ambiguity fix over the total number of epochs. As presented in Table 4, the first column gives the model schemes, whereas the first four rows represents the geometry-free ambiguity resolution using rounding, and the remaining rows represents the signals used together in geometry-based models and LAMBDA being used to determine the integers. Obviously the success probabilities of ambiguity resolution are very high for all EWLs, especially for $\Delta \Phi_{(0,1,-1)}$ with geometry-free model and $\Delta \Phi_{(1,-6,5)}$ with geometry-based model; they are almost $100 \%$ and distance-independent. However, the ambiguity resolution success probabilities for all ML and NL signals are much lower and strongly dependent on the baseline length. For instance, their success probabilities reduce from about $83 \%$ to $24 \%$ with baseline length from $53 \mathrm{~km}$ to $155 \mathrm{~km}$. Therefore, it is too hard to solve ML/NL ambiguities using geometry-free or geometry-based models without giving due consideration of systematic biases present in long baseline data.

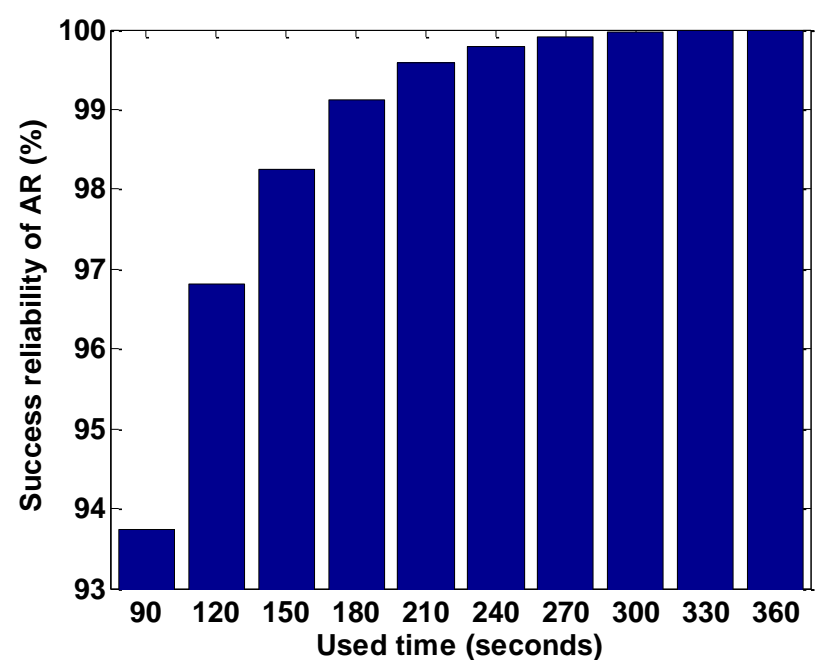

Figure 5 Success probability of ambiguity $\Delta \mathrm{N}_{1}$ versus the used time in case of $155 \mathrm{~km}$ baseline

We now assess the efficiency of the new geometry-free and ionosphere-free method for ML/NL ambiguity resolution proposed in section 4. As illustrated in Table 3, the ambiguity resolution results are equivalent for all schemes, but the random noise in cycles is enlarged. Thus, we use the $7^{\text {th }}$ scheme in Table 3 and average the float ambiguities over multiple epochs to achieve the correct ambiguities. The success probability of $\Delta \mathrm{N}_{1}$ versus the used time span for the $155 \mathrm{~km}$ baseline is illustrated in Figure 5. Apparently, the 99\% achievement can be obtained after 180 seconds and $100 \%$ after 360 seconds in the generated data set. It is emphasized that the new method is free of both ionospheric and geometric effects, and thus being very suitable for the reliable ML/NL ambiguity resolution in network-based RTK process.

\section{Concluding remarks}

We have theoretically and numerically analyzed the geometry-free and geometry-based models for TCAR for different base-rover distances. In general, the EWL ambiguity resolution is easy and can achieve $100 \%$ success probability over long distance with single epoch measurements. The difficulty is with ambiguity resolution for the ML/NL signal due to the serious effect of tropospheric biases (after the effect of ionospheric bias is largely mitigated). A new geometry-free and ionospherefree model has been introduced for reliable ML/NL ambiguity resolution over multiple epochs. The experimental results from a $155 \mathrm{~km}$ baseline have demonstrated that the new model can efficiently determine the ML/NL ambiguities over several minutes without distance constraints. This is very promising result for regional network-based RTK, where successful ambiguity resolution may take tens of minutes to hours with dual-frequency data.

Acknowledgements This work is supported by Cooperative Research Centre for Spatial Information (CRC-SI) project 1.4 for regional GNSS positioning, the National Natural Science Funds of China (Grant No. 40674003; 40874016) as well as the Key Laboratory of Advanced Engineering Surveying of SBSM (Grant No. TJES0809). The authors are very grateful to the editor-inchief Professor Alfred Leick for his constructive comments and suggestions on the manuscript.

\section{References}

Collins J. (1999) Assessment and development of a tropospheric delay model for aircraft users of the Global Positioning System, Technical Report No. 203, 
University of New Brunswick, New Brunswick, Canada.

Feng Y., Rizos C., Higgins M. (2007) Multiple carrier ambiguity resolution and performance benefits for RTK and PPP positioning services in regional areas, In: Proceedings of ION GNSS 20th International Technical Meeting of the Satellite Division, 25-28 Sept., Fort Worth, TX, pp: 668-678

Feng Y. (2008) GNSS three carrier ambiguity resolution using ionosphere-reduced virtual signals, Journal of Geodesy, 82 (12): 847-862,

Feng Y., Li B. (2008) A benefit of multiple carrier GNSS signals: regional scale network-based RTK with doubled inter-station distances, Journal of Spatial Science, 53(1):135-147

Forssell B., Martin-Neira M., Harris R. (1997) Carrier phase ambiguity resolution in GNSS-2, In: Proceedings of ION GPS-97, 16-19 Sept., Kansas City, pp: 17271736

Hatch R., Jung J., Enge P., Pervan B. (2000) Civilian GPS: the benefits if three frequencies, GPS Solutions, 3(4):1-9

Henkel P, Cünther C. (2007) Integrity analysis of cascaded integer resolution with decorrelation transformations, In: Proceedings of the Institute of Navigation, National Technical Meeting, San Diego

Ji S., Chen W., Zhao C., Ding X., Chen Y. (2007) Single epoch ambiguity resolution for Galileo with the CAR and LAMBDA methods, GPS Solutions, 11:259-268

Leick A. (2004) GPS satellite surveying, $3^{\text {rd }}$ edn. Wiley, New York

Li B. (2008) Generation of third code and phase signals based on dual-frequency GPS measurements, ION GNSS 2008, 16-19 Sept., Savannah GA, pp: 2820 2830

Richert T., EI-Sheimy N. (2007) Optimal linear combinations of triple frequency carrier phase data from future global navigation systems, GPS Solutions, 11:11-19

Vollath U., Birnbach S., Landau H. (1998) Analysis of three carrier ambiguity resolution(TCAR) technique for precise relative positioning in GNSS-2, In: Proceedings of ION GPS98, 15-18 Sept., pp: 417-426 